\title{
¿Desaparecerán los Tour Operadores? El papel de los in- termediarios en la distribución turística: Análisis del caso de Tenerife
}

\author{
Davinia Flores Alberto \\ Lisandra Salazar Niebla ${ }^{\mathrm{i}}$ \\ Manuel Ángel Santana Turégano ${ }^{\mathrm{ii}}$
}

Universidad de La Laguna (España)

\begin{abstract}
Resumen: En los últimos años diversos autores han planteado el fin de la intermediación turística. Dado que Internet permite la comunicación efectiva entre proveedores de servicios turísticos y clientes geográficamente distantes, cada vez sería menor el porcentaje de personas que recurrirían a intermediarios para organizar sus viajes. Otros autores afirman que el impacto de las nuevas tecnologías puede no ser la desintermediación, sino el cambio en la intermediación, en función de factores como la cercanía cultural entre turista y destino. En este trabajo se ha analizado el caso de Tenerife (España) y se ha encontrado que el uso de los intermediarios aún sigue siendo ampliamente mayoritario. Por ello, se plantea que factores asociados a la estructura de los mercados turísticos, frecuentemente poco considerados, pueden estar influyendo en la configuración de la distribución turística.
\end{abstract}

Palabras clave: Touroperadores; Intermediación; Paquete turístico; Turismo de sol y playa; Islas Canarias; Tenerife.

Title: Tour operators will disappear? The role of intermediaries in the tourism distribution: the case of Tenerife

\begin{abstract}
In the later years several authors have posed the idea of the end of tourism operators. Since the internet allows an effective communication between providers of tourism services and clients that are geographically distant, fewer people will use such agents to organize their travels. Others authors have suggested that rather than a des- intermediation, the impact of IT in the tourism industry would be that of the change in intermediation, depending upon factors such as the cultural closeness between tourists and destinations. This paper analyzes the case of Tenerife (Spain), finding that the use of tour operators is still the most common trend in the industry. Therefore, it is suggested that factors related with the structure of tourism markets might be influencing tourism distribution
\end{abstract}

Keywords: Tour Operators; Intermediation; Package tour; Sea and Sun tourism; Canary Islands; Tenerife.

i Máster en Dirección y Planificación del Turismo, Universidad de La Laguna.

ii Doctor en Sociología, Profesor del Departamento de Sociología de la Universidad de La Laguna. E-mail: masantur@ull.es 


\section{Introducción}

Con la expansión del uso de Internet por parte de los turistas, tanto para la búsqueda de información como para la contratación de servicios de alojamiento y transporte, se están dando grandes cambios en la estructura del canal de distribución turístico, lo que pondría en entredicho el papel desempeñado por algunos de los actores del mismo. Este comportamiento podría favorecer un proceso de desintermediación (Doherty, EllisChadwick y Hart, 1999), en el cual las agencias de viajes minoristas podrían ser las más perjudicadas, tanto por las implicaciones que el desarrollo de la red conlleva como por el número de agencias de viajes minoristas que hay. Sin embargo, otros autores plantean que esta desintermediación afecta tan sólo a ciertos segmentos del mercado turístico. Estos autores plantean que la forma en que se adquieren los productos turísticos, ya sea directamente o a través de intermediarios, se relaciona con el tipo de viaje del que se trate, de forma que para algunos tipos de viaje el uso de intermediarios sería mayoritario y minoritario para otros (Eymann y Ronning, 1992). Así, el uso de las nuevas tecnologías (que facilitan la compra directa) tiene un mayor impacto en la contratación de productos turísticos de baja implicación y poco especializados, es decir, un individuo tendrá una predisposición mayor a reservar un vuelo a través de Internet que a adquirir directamente un paquete turístico todo incluido (Falkenstein, 1997). En cambio, la compra de productos turísticos a través de agencias de viajes se asocia a productos de alta complejidad (Mak y Moncur, 1980; Sheldon y Mak, 1987; Esteban et al., 2000), como los viajes internacionales, debido a la reducción de la incertidumbre que estos conllevan, y al ahorro de tiempo en la organización de estos viajes con múltiples componentes (como transporte, alojamiento, reservas, etc.). Sheldon y Mak (1987) plantearon en su estudio, realizado hace más de veinte años, que los viajes con un destino itinerante tienden también a favorecer la contratación de intermediarios, ya que permite agregar más eficientemente todos los elementos del producto.

La literatura que avala la tesis de la desintermediación es abundante. Benjamin y Wigand $(1995,1997)$ defienden que el comercio electrónico puede reducir la presencia de los intermediarios en la cadena de valor. Otros autores como Quelch y Klein (1997) o Buhalis (1999) plantean que la desintermediación en el sector turístico surgirá como consecuencia de la eliminación de los costes de transacción que permiten las Nuevas Tecnologías de la Información y las Comunicaciones (en adelante, NTIC). Sin embargo, otros autores, como Hawkins, Mansell y Steinmuller (1998) consideran que no se dará tal desintermediación sino cambios en la intermediación, ya que los intermediarios cumplen funciones importantes de acuerdo con la teoría del marketing. Desde los inicios de la industria turística, tal y como en la actualidad se la conoce, los intermediarios turísticos han desempeñado numerosas e importantes funciones. Permiten reducir el número de contactos y transacciones para agregar una experiencia o producto turístico, ajustar la oferta a la demanda, crean un "surtido" o abanico de productos turísticos de cara al consumidor, facilitan las actividades de marketing para los proveedores de servicios (empresas alojativas), pueden facilitar la financiación de las empresas intermediadas (fenómeno que ha tenido históricamente mucha importancia con los hoteles en destinos como Canarias y en general España) y por último ofrecen servicios adicionales al consumidor, desde la tramitación de visados o seguros a servicios de transporte o transfer (Alcázar Martínez, 2002). Sin embargo, desde el punto de vista de los destinos turísticos, los intermediarios han sido vistos en muchos casos con recelos. Así, frecuentemente suele plantearse que los tour operadores derivan parte de los beneficios de la actividad turística fuera de las zonas receptoras, y que pueden desviar los flujos de turistas hacia unos u otros destinos en función de sus intereses. Desde el punto de vista empresarial, se ha planteado que las presiones de los TO y las AAVV pueden llegar a estrangular la operatividad de las empresas (Parra López, Melchior Navarro y Domínguez Ramos, 2003)

En cualquier caso, comenzada ya la segunda década del siglo XXI, y habiendo transcurrido cierto tiempo desde la generalización de las Nuevas tecnologías de la Información y las Comunicaciones (NTIC) en el sector turístico, creemos que pueden hacerse algunas generalizaciones acerca del impacto de las mismas en la intermediación. Y la principal de ellas es que parece haber importantes diferencias, en función del tipo de destinos y de productos turísticos, en la manera en que el desarrollo de las TIC ha impactado a la industria. A nivel internacional, Parra López, Melchior Navarro y Domínguez Ramos citaban datos del European Travel Monitor que estimaban que a principios de siglo (año 2001) en un $77 \%$ de los viajes realizados desde el Reino Unido y un 54\% de los realizados desde Alemania se habían utilizado intermediarios (Tour operadores o agencias de viaje, paquetes flexibles o estandarizados). Si comparamos estos datos de principios de la década del 2000 con los que la Encuesta del Gasto Turístico provee para España en 2009 parecería que efectivamente el rol de la intermediación en la industria turística ha disminuido enormemente. De acuerdo con dicha encuesta podría estimarse que un $30 \%$ de los turistas que visitaron España en ese 
año contrataron un paquete turístico, si bien existen importantísimas diferencias. Así, en función del país de origen de los turistas, parece que efectivamente aquellos más lejanos culturalmente tienden a utilizar los paquetes turísticos en mayor medida: más de un $60 \%$ de los japoneses, por cantidades cercanas al 40\% en los Países Nórdicos o Alemania, e inferiores al 10\% para nuestros países limítrofes, Francia y Portugal. Si atendemos a las comunidades autónomas de destino, el paquete turístico sigue siendo ampliamente mayoritario en Canarias y Baleares (cerca de un 70\% de los turistas que visitan las islas, siempre en relación a los datos de EGATUR). En cualquier caso, la propensión a utilizar el paquete turístico tiene mucho que ver con el tipo y el motivo del viaje, así como con la zona geográfica que se analiza, de forma que el paquete turístico comercializado por un touroperador ha sido la forma predominante en la organización de los viajes vacacionales de sol y playa de los europeos en la segunda mitad del siglo XX (Cavlek, 2005). Tomando como referencia el mercado emisor español, si bien las conclusiones parecen generalizables a otros mercados similares, Rodríguez González y Molina Molina (2009) plantean que algunos tipos de viajes en segmentos más especializados como los viajes de negocios, el turismo rural o las escapadas cortas (short breaks) son contratados en menor medida a través de paquetes.

En este estudio se analizarán los datos de la Encuesta de Satisfacción realizada por Turismo de Tenerife en 2009 para analizar el uso de los distintos canales de distribución por parte de los turistas que visitan un destino maduro de sol y playa como Tenerife. Se analizaron los datos en función de la tipología del alojamiento, del origen de los turistas y de las zonas de estancia. La conclusión principal del estudio es que los turistas que visitan Tenerife siguen optando, en un porcentaje cercano al 70\%, por utilizar intermediarios (Touroperadores o Agencias de Viaje), lo que implicaría que en los últimos años no se habría realizado un avance significativo en la línea de la desintermediación. Para el destino analizado, el uso de intermediarios es fundamental en el grueso de la oferta turística, concentrándose la contratación directa en fórmulas de viaje relativamente marginales (Time Sharing, Casas Particulares) y que por su propia organización son ajenas al concepto de la tour operación. De todo ello se deduce la existencia de ventajas de la intermediación respecto a la comercialización directa, tanto para el turista ( economías de escala) como para la oferta (capacidad de planificación de la producción), y por lo tanto, pese a lo que planteaban algunos autores, no parece plausible, al menos para destinos como el analizado, en el corto y medio plazo, la desintermediación. Para tratar todo esto, el resto del artículo se organizará de la manera siguiente. En primer lugar, explicaremos las cuestiones metodológicas del estudio realizado. En segundo lugar, expondremos los principales resultados del mismo. Por último, pasaremos a tratar algunas conclusiones y las implicaciones de las mismas.

\section{Ámbito de la investigación}

Para analizar cómo los turistas contratan sus viajes y si se usan o no los intermediarios (Touroperadores y Agencias de Viaje) hemos optado en este estudio por analizar los mecanismos de contratación que utilizan los turistas que visitan la isla de Tenerife (Islas Canarias, España). La investigación se centra así en un mercado receptor (un destino) y no pretende por tanto ser representativa de la evolución del turismo actual sino de la evolución de determinados tipos de destinos. En concreto, de los destinos turísticos de sol y playa que, de acuerdo con el ciclo de vida de las áreas turísticas (Butler, 2006) pueden considerarse maduros. Estamos hablando de destinos de sol y playa que llevan ya un largo tiempo en el mercado, y que se enfrentan a retos de reestructuración (Agarwall, 2002) para reposicionarse en el mismo. Además, y ésta es no es una cuestión baladí, pensamos que éstas conclusiones se restringen sobre todo a aquellos destinos que, por distintos motivos, tienen turistas que les visitan mayoritariamente por vía aérea ${ }^{1}$. En cuanto al uso de la intermediación en el destino, si atendemos al caso de Canarias, Parra López, Melchior Navarro y Domínguez Ramos (2003), usando datos de la Encuesta del Gasto Turístico del Gobierno de Canarias muestran que a principios de siglo (años 2001 y 2002) un 70\% de los turistas que visitaban las islas utilizaban intermediarios turísticos (T.O), si bien esta cifra era algo inferior para los británicos y mayor para los alemanes, con lo cual podría pensarse que apenas se habían producido cambios significativos al respecto.

\section{Planteamientos metodológicos del estudio}

Como ya hemos dicho, el análisis de un destino para estudiar la evolución de la intermediación, a pesar del inconveniente de no poder generalizar los datos para el conjunto de la industria turística mundial en la actualidad, presenta indudables ventajas. En primer lugar, la fiabilidad y robustez, tanto teórica como estadística, de los datos así conseguidos. Los estudios sobre los mercados emisores suelen preguntar a un conjunto de ciudadanos (de un país o región) que ha viajado durante 
un período de referencia cómo organiza sus viajes. Ello puede dar lugar a todo tipo de sesgos. Así por ejemplo, se llega a veces a estimaciones del tipo "El porcentaje de los viajes que los alemanes realizaban a través de un TO ha caído de un 75 a un 35\% entre 1970 y 2010". Pues bien, este tipo de estudios tiene distintos problemas que pensamos que pasan fundamentalmente por tres tipos de posibles sesgos. En primer lugar, el sesgo lingüístico o conceptual: no todo el mundo entiende lo que se le pregunta cuando se le interroga acerca de si han usado o no Tour Operadores. Las personas que no pertenecen a la industria turística generalmente no conocen el funcionamiento real de la misma. Así, pueden contestar que organizan un viaje por su cuenta, sin intermediarios, cuando entran en Internet y reservan un alojamiento a través de páginas como "booking.com". Y esto, evidentemente, resulta del todo inexacto desde el punto de vista de la industria turística (han utilizado un intermediario, aunque sea virtual). En segundo lugar, el sesgo de memoria: la forma de realizar estas encuestas hace que sea muy engorroso recabar datos cuando los sujetos realizan más de un viaje al año. De manera que si clasificamos a un turista como "turista que no usa intermediarios" en base a su respuesta, ello puede contener numerosas inexactitudes. Quizá la persona que contesta que en vacaciones reservó por su cuenta olvida aquella excursión que realizó con su equipo deportivo, parroquia o compañeros de trabajo y que correspondía perfectamente a la definición del "paquete turístico". Por último, está el sesgo de la deseabilidad social (común por lo demás a todas las encuestas). En una sociedad que ensalza la individualidad y el uso de las nuevas tecnologías, es bastante razonable pensar que las personas prefieren declararse "viajeros independientes que utilizan Internet" que no "viajeros masivos que compran un paquete en que todo les viene dado".

Para evitar todos estos inconvenientes aquí se ha optado por una aproximación metodológica bastante distinta, que aunque analiza los turistas (la demanda) centra más su importancia en la oferta de lo que suele ser habitual. Así, se han utilizado los datos de una encuesta sobre satisfacción con la visita a la isla realizada por el Cabildo de Tenerife. La encuesta, con una muestra total de 5.000 turistas y realizada en los dos aeropuertos de la isla a lo largo del año 2009, era representativa, a un nivel de confianza del $95 \%$ y margen de error del 5\%, del conjunto de los turistas que visitaron la isla en ese año. Para cada turista se recogía información del establecimiento en que se alojaba en la isla (todos los turistas han de pernoctar en algún lugar) y de la fórmula utilizada para contratar éste. Se obtuvo así información acerca de los mecanismos utilizados para reservar el alojamiento por 4.556 turistas, que resultaban representativos, siempre de acuerdo a las condiciones anteriormente indicadas, de los más de 4,5 millones de personas que a lo largo de 2009 utilizaron los establecimientos alojativos (hotelero y extrahoteleros) de Tenerife (Datos de Turismo de Tenerife, 2009). Para la cuestión que aquí nos interesa, la de si el desarrollo de las nuevas tecnologías provoca la desintermediación o un cambio en la intermediación en el sector turístico, creemos que esta manera de recabar los datos es especialmente pertinente. Al igual que muchas encuestas específicas al respecto, la encuesta que se utilizó para recabar los datos desglosaba los mecanismos de reserva del alojamiento en función del canal utilizado (presencial, teléfono, fax o Internet). Desde el punto de vista que aquí nos interesa, lo importante en la reserva no es el canal utilizado, sino si existen o no intermediarios, cuántos y de qué tipo. Así por ejemplo, lo relevante no sería si un turista británico que se alojó durante una semana del verano de 2009 en Costa Adeje (Sur de Tenerife) realizó la reserva en una pequeña agencia de viajes de su barrio, en una oficina franquicia de un Tour Operador o directamente en la página web de éste. Sino si utilizó un tipo de intermediario (el Tour Operador) que ofrecía un paquete compuesto por transporte y alojamiento. En el caso de que utilizase un intermediario para contratar tan sólo el alojamiento, y el transporte lo contratara directamente al proveedor del mismo, poco importaría si éste fuera on-line o se trata de una agencia de viajes física. Por último, aunque no por ello menos importante, el análisis de la intermediación a través de la fórmula de contratación del alojamiento en un destino determinado también presenta otra ventaja, la de permitir hacerse una idea más real de la articulación actual de la intermediación turística.

Una de las tendencias más importantes en el turismo en los últimos años ha sido la irrupción de las aerolíneas de bajo coste (Low Cost), que han tenido un significativo impacto en España (véase al respecto Ivars Baidal, 2006). Estas compañías, que venden la práctica totalidad de sus billetes a través de Internet (si bien existen agencias de viajes virtuales que venden también sus billetes ${ }^{2}$ ), ofrecen en su página web la posibilidad de contratar o reservar también, cuando se compra un billete, otros servicios como alojamiento, alquiler de vehículos, transporte desde los aeropuertos a las ciudades (airport transfer) o incluso servicios de telefonía móvil para la duración de la estancia en otros países. Todo ello puede generar una importante confusión tanto en el consumidor final como en los mismos estudiosos del turismo. Un consumidor no muy informado puede tener la sensación, al contratar su viaje a través de la web 
de una low cost, de ser un "viajero independiente" que elige su vuelo y después elige entre un amplio abanico de alojamientos u opciones de transporte. Un académico podría pensar que estamos ante el auge de los "paquetes dinámicos", realizados a la medida del consumidor (sobre las tecnologías web y las posibilidades de los paquetes dinámicos puede verse Cardoso y Lange, 2007 o Cardoso, 2006). Lo que es indudable en cualquier caso es que asistimos a procesos de cambios en la intermediación turística, en los cuales el cambio en los mecanismos de contratación de uno de los componentes del turismo (el transporte) puede ir unido o no a cambios en la contratación de otros componentes como el alojamiento. Por lo tanto, pensamos que el análisis de cómo los turistas contratan un solo servicio, en este caso el alojamiento, presenta indudables ventajas desde el punto de vista metodológico al del análisis de la contratación del conjunto de la experiencia turística ${ }^{3}$. La siguiente tabla resume las características principales del planteamiento metodológico

\begin{tabular}{|l|l|}
\hline Técnica & Encuesta \\
\hline Muestra & 4.556 turistas \\
\hline Universo & Turistas que se alojaron en Tenerife en 2009 \\
\hline Cuestión analizada & Cómo reservaron su alojamiento \\
\hline Opciones & Intermediarios/directo (no importa mecanismo)
\end{tabular}

\section{Resultados de la investigación}

Una vez recabados los datos a través de la encuesta y categorizados de acuerdo a la manera ya señalada, es decir, analizando si los turistas utilizaban un intermediario para reservar el alojamiento y no los medios materiales utilizados para realizar la reserva los datos fueron analizados. Para ello se utilizó el paquete estadístico SPSS, llevándose a cabo un análisis centrado en la estadística descriptiva, con pequeñas incursiones en el análisis de datos (tablas de contingencia) lo cual resultaba coherente con los objetivos del trabajo. Las conclusiones principales del análisis señalan que los turistas que visitaron Tenerife durante 2009 usaron mayoritariamente intermediarios, con sólo una cuarta parte de los mismos utilizando la contratación directa. Esto es así para todas las categorías de alojamiento utilizados, si bien parece existir una tendencia en el sentido de que los establecimientos de menor categoría son los que usan en mayor medida la contratación directa. Tampoco se detectaron diferencias significativas en función del municipio de la isla en que los turistas disfrutaron su estancia. Por último, aunque existe una cierta tenden- cia a que los turistas repetidores usen más la contratación directa que los turistas que acuden por primera vez a la isla, resulta significativo y digno de reseñar que los turistas que ya conocen la isla de visitas anteriores siguen utilizando mayoritariamente los intermediarios a la hora de reservar su alojamiento, si bien esto puede estar influido por su mayor edad ${ }^{4}$. A continuación pasamos a exponer los datos acerca del uso de intermediarios en la contratación del alojamiento en función de las distintas variables utilizadas.

En primer lugar, la principal aportación de los datos de la investigación es que, para el año 2009, la mayoría de los establecimientos alojativos de Tenerife no se relacionaban de forma directa con sus clientes, sino a través de intermediarios. Así, del total de 4.556 turistas de los cuales se recabaron datos acerca de cómo reservaron su alojamiento, sólo el 25,44\% contrató su estancia directamente en el alojamiento utilizado, por medio de los distintos canales posibles (Internet, Fax, Teléfono o en la propia recepción, se entiende que para turistas re-

petidores). Un 55,95\% realizó la reserva a través de una Agencia de Viajes, y un 18,61\% realizó la reserva a través de un Tour Operador ${ }^{5}$.

Como ya hemos dicho, la utilización de intermediarios es mayoritaria también en la mayoría de categorías y tipos de alojamiento. Para desglosar el análisis en función del tipo y categoría del

establecimiento es necesario en primer lugar reseñar que el alojamiento hotelero de cuatro estrellas es el más difundido en la isla de Tenerife. De acuerdo a los datos de Turismo de Tenerife, de los 4,7 millones de turistas alojados en la isla en 2009 , un $59,08 \%$ se alojaron en hoteles, y el 60,99\% de éstos (es decir, prácticamente 1,7 millones de turistas) en hoteles de cuatro estrellas. Es decir, que tan sólo en los hoteles de 4 estrellas se alojaron más del $36 \%$ de los turistas que visitaron la isla en ese año. Le siguen en importancia los hoteles de 3 (21\% del total de turistas alojados en hoteles en la isla) y cinco estrellas (13\%), totalizando entre ambos prácticamente un millón de turistas (lamentablemente, la fuente, Turismo de Tenerife, no desglosa los turistas alojados en establecimientos extrahoteleros según su categoría (Datos extraídos de Turismo de Tenerife, 2009). Pues bien, de entre todas las categorías consideradas $^{6}$ la contratación directa sólo era mayoritaria en los hoteles de 1 y 2 estrellas (70\%), en las casas/apartamentos privados $(67,7 \%)$ y en los alojamientos de Time Sharing (68\%) siendo prácticamente equitativa la distribución en el caso de los apartamentos de una llave (un 50\% se reservaron directamente, un 16,1\% a través de TTOO y un 29\% a través de agencias de viaje). 
Pese a que la significatividad estadística de estos datos es menor que la de otras categorías, debido a la reducida participación de estos establecimientos en el alojamiento de turistas en Tenerife, estos parecen señalar un par de tendencias interesantes. Por una parte, que la reserva del alojamiento mediante intermediarios se relaciona altamente con el uso del "Paquete Turístico", aún ampliamente predominante. Es por tanto en aquellas categorías de alojamiento que se comercializan en menor medida por los canales habituales donde se da mayor porcentaje de reservas directas. En realidad, el hecho de que el alojamiento en "casas particulares" se contrate directamente es una aparente contradicción en los términos que necesita de una cierta explicación. En la encuesta se ofrece a los turistas la posibilidad, al interrogarles sobre su alojamiento, de responder que habían pasado sus vacaciones en una "casa o apartamento privados", y 18 preguntas después, cuando el turista ha olvidado quizá lo que había contestado, se le preguntaba cómo había contratado dicho alojamiento. La categoría "casa o apartamento privado" es común en las encuestas realizadas por el Gobierno de Canarias para el conjunto del archipiélago, así como en otros lugares de España. En teoría, entrarían dentro de esta categoría aquellos turistas que se alojan, bien en una segunda residencia, bien en la casa o en lo segunda residencia de amigos o familiares, que en principio les habría facilitado gratuitamente. En realidad, esta situación corresponde a una situación que se ha ido dando de forma cada vez más generalizada a partir de los años 2002-03, de acuerdo con la información recabada al realizar entrevistas exploratorias previas a la realización de este trabajo. A partir de esa época, diversos factores como la fortaleza de la libra frente al euro, algunas de las políticas aplicadas en el Reino Unido de forma diferencial al resto de Europa, la escasez de entornos "vacacionales" más cercanos y la existencia de una tupida red de conexiones aéreas gracias a las aerolíneas Low Cost han llevado a la generalización del turismo residencial en muchas áreas del mediterráneo (sobre este fenómeno puede verse Huete, 2009). Esto se ha concretado en que un gran número de ciudadanos del norte de Europa, fundamentalmente británicos, han adquirido en propiedad apartamentos y casas vacacionales, entre otros destinos, en Tenerife (y es significativo señalar que ésta es la isla más importante de Canarias para el turismo británico, a diferencia de otras donde el alemán y el nórdico tienen más importancia). Estos ciudadanos británicos alquilan sus apartamentos vacacionales, durante los períodos en que no los utilizan, a otros conciudadanos, mediante distintos mecanismos más o menos informales ${ }^{7}$. En definitiva, y para los objetivos que en este trabajo nos ocupa, es importante señalar que parte de los turistas de Tenerife que reservan su alojamiento directamente son aquellos que utilizan este tipo de alojamiento que, al margen de los grandes circuitos turísticos, quedan también, de acuerdo con la legislación vigente en Canarias, en una situación un tanto ilegal o al menos alegal.

En cuanto a otra de las variables que influyen en el uso de intermediarios o no, pasamos a considerar a continuación la familiaridad con el destino. Si tenemos en cuenta el número de visitas anteriores, y pese a que la forma en que se recogían los datos no permitía establecer matices según el número de visitas realizadas, podemos afirmar que, de acuerdo a lo que se podría esperar los turistas que repiten visita usan más la contratación directa que los que acuden a la isla por primera vez (de los repetidores recurren a la contratación directa un $30,91 \%$, por un 18,2 de los que visitan la isla por primera vez). Son menos los que recurren a Agencias de Viajes (48\% de los repetidores las utilizan para contratar su alojamiento por un 64\% de los "primerizos") pero, sorprendentemente, el porcentaje de los que reservan a través de un touroperador es incluso ligeramente superior $(20,5 \%)$ entre los repetidores que entre quienes visitan la isla por primera vez. Por último, se realizó un análisis también en función de los municipios de residencia de los turistas en la isla, que no se detallará aquí porque la única diferencia reseñable era que en los municipios más turísticos se reforzaba la pauta aquí señalada (contratación a través de intermediarios) y que ésta se presentaba de manera más débil en los municipios con menor importancia turística.

\section{Conclusiones e implicaciones de la investigación}

La principal aportación de la investigación es que, una vez revisada la evidencia empírica para el caso de estudio (Isla de Tenerife, año 2009), el uso de intermediarios por parte de los turistas a la hora de contratar un alojamiento sigue siendo ampliamente mayoritario (en torno a un $75 \%$ ) y además, en función de los datos disponibles, no parece haberse reducido de forma muy importante en el último decenio. Aunque posteriormente detallaremos las principales conclusiones de la investigación, ofrecemos aquí de forma sintética una visión panorámica de las mismas:

1. La intermediación sigue siendo ampliamente mayoritaria en Tenerife. El $75 \%$ de los turistas que visitan el destino reservan el alojamiento a través de un intermediario, pese al crecimiento de las tecnologías de la información y a la creciente familiaridad de los turistas con la isla.

2. La intermediación es producto de la estructura del 
mercado y no solo de la tecnología, por lo que no cabe pensar que el cambio tecnológico la elimine.

3. En cualquier caso, lo que sí se da son cambios en los agentes que intermedian. Es importante que las Administraciones Públicas tengan esto en cuenta pues sus actuaciones pueden favorecer a unos actores y perjudicar a otros.

Estas conclusiones parecen generalizables para destinos similares, de manera que parece que, al menos si hablamos de destinos de sol y playa a los que se accede fundamentalmente por vía aérea, el impacto de las Nuevas Tecnologías de la Información y las Comunicaciones (NTICs) en la intermediación turística ha sido más el de provocar un cambio en la misma (una re- intermediación) que su desaparición, la hipotética desintermediación que planteaban autores como Quelch y Klein (1997) o Buhalis (1999). La re-intermediación, tal y como aquí la planteamos, hace referencia a dos fenómenos relacionados. Por una parte, que algunas modalidades de turismo pasarían a estar más o menos intermediadas. Por otra, que los intermediarios en concreto, y los mecanismos, pueden haber cambiado y continuar cambiando.

En primer lugar, los datos que hemos obtenido señalan una tendencia que coincide con la detectada por otro tipo de estudios, el mantenimiento de la intermediación en destinos que podríamos denominar "vacacionales, de sol y playa y con un importante componente climático". Sin embargo, estudios más generales, como los datos de Frontur ya señalados anteriormente, parecen detectar que en otros segmentos de la industria turística, como los viajes de negocios, las escapadas cortas o los viajes de naturaleza al interior, el uso de intermediarios a la hora de contratar alojamientos podría ser la excepción más que la norma, de acuerdo a lo que señalan Rodríguez González y Molina Molina (2009). Por lo tanto, cabe hablar de un cambio en la intermediación en la industria turística en la medida en que en algunos subsectores de la misma la intermediación tiende a desaparecer mientras que permanece constante en otros, como el turismo vacacional que aquí hemos analizado.

Pero la re- intermediación puede interpretarse también como un cambio en los actores dentro de cada situación particular. Es decir, para el caso del turismo vacacional se mantiene el hecho de que la intermediación sigue siendo la fórmula mayoritaria, pero cambian tanto los actores principales como los mecanismos de relación. Como ya hemos visto en el caso de las aerolíneas de bajo coste que comercializan alojamiento o los ciudadanos británicos particulares que alquilan sus apartamentos en Tenerife en Webs específicamente dedicadas a ello. Y ello implica diferentes repartos tanto en la creación de valor como en la asunción de riesgos en los diferen- tes actores que intervienen en la cadena global de valor de la industria turística. Los autores que planteaban, hace ya casi diez años, la tesis de la intermediación se basaban fundamentalmente en las potencialidades para el cambio que presentaban las NTICs. Partiendo de la idea de que la investigación en Turismo ha de anclarse sólidamente en los marcos teóricos de las disciplinas de referencia (Santana Turégano, 2009), a continuación intentaremos avanzar algunas explicaciones de este fenómeno a partir de la teoría económica y de la nueva sociología económica, lo cual nos permitirá señalar las implicaciones teóricas y para la gestión del estudio.

Tal y como aquí lo planteamos, el hecho de que en los destinos vacacionales/climáticos de sol y playa la relación entre los turistas y los establecimientos alojativos se haga fundamentalmente a través de intermediarios tiene que ver con la estructura de los mercados turísticos. Los turistas prefieren contratar un determinado hotel a través de un paquete con un TO que adquirir de forma separada el vuelo y el alojamiento, sencillamente, porque en muchos casos les resulta más económico. Como ya hemos señalado, se trata de destinos a los cuales se accede fundamentalmente por avión, ya sean destinos insulares o no. Y esto es algo implícito a su propio carácter de "destinos climáticos": el avión es el único medio de transporte que permite, en pocas horas, pasar de entornos de climas fríos a otros de climas más cálidos. Pues bien, desde la teoría económica más clásica de la empresa ya se ha establecido que podría calcularse el número de empresas que pueden operar en un mercado, en función de los costes medios y las posibilidades de producción. Lo cual nos lleva a constatar un hecho evidente: la estructura del mercado del transporte aéreo entre los destinos turísticos climáticos y sus mercados emisores es siempre la de un oligopolio. Es decir, por poner un ejemplo, en el caso de Tenerife existen sólo unas pocas compañías aéreas que comunican la isla con los principales aeropuertos británicos o alemanes. Nos encontramos por tanto con una estructura dual del mercado turístico. En una parte del mismo (el subsector alojativo) es posible la competencia de mercado. En otra (el subsector del transporte) lo que se da es un oligopolio. En definitiva, el transporte (aéreo) se convierte en una especie de cuello de botella para que los establecimientos alojativos accedan a sus clientes: no importa cómo decidan comunicarse con ellos, al final éstos habrán de llegar a la isla a través de alguno de los 4 ó 5 operadores aéreos existentes. Parra López, Melchior Navarro y Ramos Domínguez (2003) planteaban que los intermediarios podían "estrangular" la operatividad de las empresas alojativas. Evidentemente, lo que parece claro es que están en disposición de establecer las condiciones 
y poseen un gran poder de mercado.

¿Sería posible otra estructura del mercado turístico? En los últimos años, la política turística de muchos destinos, entre ellos Canarias y Tenerife, ha ido en la línea de intentar cambiar la situación. Dado que existe una división internacional del trabajo en la industria turística, de manera que las empresas alojativas suelen estar radicadas en los lugares de destino y los TO y las líneas aéreas en los lugares de origen de los turistas, los gestores de los destinos han intentado reconducir la situación. La idea subyacente a estas políticas es que si se consigue una mejor situación para las empresas alojativas, la economía (y la sociedad) de los destinos se vería beneficiada. Y para modificar la situación se ha intentado mejorar las conexiones aéreas del destino, atrayendo aerolíneas, en muchos casos de bajo coste, y en ocasiones a costa de subvenciones públicas más o menos encubiertas. Los datos aquí presentados parecen señalar que a pesar de los esfuerzos realizados no se ha conseguido una menor presencia de los intermediarios en la distribución turística.

Un análisis de los procesos que llevan a cabo un turista al reservar un vuelo con una compañía de bajo coste ilustra los cambios acaecidos en la relación entre los agentes del sector turístico. Un turista que compra un billete a una Low Cost ha de abonarlo en el momento de realizar la reserva. La compañía elimina así incertidumbres y adquiere recursos monetarios. Aunque el turista se ponga enfermo o decida finalmente no realizar el viaje, la compañía ya ha cobrado el dinero y puede planificar la producción en base a ello. Ahora bien, el mismo turista, si utiliza los enlaces facilitados por la línea aérea para reservar su alojamiento, realizará tan sólo una reserva que no pagará, pudiendo anularla, en función de la compañía de que se trate, hasta 24 horas antes sin que le suponga ningún coste. La posible ganancia del establecimiento alojativo ante esta "desintermediación" sería que, al no tener que pagar comisiones al Tour Operador podría obtener una parte mayor, o incluso la totalidad, de lo que el turista paga por el alojamiento. Sin embargo, existe también una pérdida: una mayor incertidumbre, lo que puede implicar importantes problemas a la hora de planificar la empresa. Y no olvidemos que, en la situación tradicional, mediante los contratos entre TTOO y establecimientos alojativos éstos podían renunciar a un mayor beneficio a cambio de una mayor previsibilidad mediante los contratos en garantía. Parece lógico pensar que la estructura de costes de la empresa influirá en la adopción de una u otra estrategia. Evidentemente, y esto es una de las implicaciones del trabajo para futuras investigaciones, parece necesario abordar este tipo de estudios desde el punto de vista de la oferta. Es decir, son necesarios estudios que, para un destino concreto, analicen cómo están comercializando sus plazas los establecimientos alojativos, y si existen diferencias entre éstos en función de variables como su situación, antigüedad, estilo de gestión, tamaño, pertenencia a cadenas u otras. Evidentemente, somos conscientes de las dificultades para hacer estudios de este tipo, pero pensamos que podrían aportar una información muy importante.

Otro ámbito de interés para continuar la investigación pasaría por los nuevos desarrollo en Sociología Económica, con conceptos claves como los de "cadenas globales de valor" (global value chain, Gereffi, 2005). Desde este enfoque, lo interesante para un destino turístico sería que las relaciones entre los establecimientos alojativos (pertenecientes al destino) y los touroperadores (generalmente externos al mismo) se pudieran llevar a cabo mediante los cauces más convenientes para el destino. Así, y utilizando la jerga propia de este enfoque (para una aplicación del mismo al turismo véase Santana Turégano, 2009), dado que parece imposible que las cadenas de valor adopten formas propias de mercado, por la posición de poder de quienes controlan el transporte aéreo, lo deseable sería que éstas se configuraran como "cadenas de valor modular o relacional", en las que tanto los operadores aéreos como los establecimientos alojativos y de otro tipo aporten valor a la experiencia turística. La tendencia en la actualidad, con la profusión de las líneas aéreas de bajo coste, parece ir en la línea de que sean éstas las que controlen la parte más importante del proceso de creación de valor en el negocio turístico (al fin y al cabo los turistas no se pueden alojar en destinos como Tenerife si un avión no les traslada hasta allí).

Por último, pero no por ello menos importante, creemos que, más allá de las implicaciones teóricas de estas conclusiones, éstas resultan importantes también para la gestión de los destinos turísticos. ¿Han de apoyar los poderes públicos la "desintermediación", bajo el supuesto de que ésta permitirá a las empresas alojativas (las del destino) quedarse con una parte mayor de la riqueza generada por el destino turístico y que por lo tanto ello repercutirá en el bienestar económico y social del mismo? La evidencia aportada en este estudio parece señalar que la intermediación, más que desaparecer, cambia. Por lo tanto, no parece tan clara la idea de que restando peso a los intermediarios tradicionales (tour operadores) logren los destinos una mejor participación en los beneficios de la industria turística. Pueden desaparecer unos intermediarios, pero aparecen otros nuevos: centrales de reserva de alojamiento en Internet, enlaces en las webs de los Tour Operadores, Agencias 
de Viajes Virtuales, etc. Por lo tanto, es importante la relación con estos nuevos intermediarios, más que pensar en la des-intermediación, que se da sólo en algunos casos muy concretos y relacionada más con cadenas que con establecimientos particulares. De acuerdo a lo señalado por Alcázar Martínez (2002) los intermediarios efectivamente realizan una función para el turista, no son meros "comisionistas" que se aprovechan de su posición de poder, como a veces son vistos. Una de estas funciones fundamentales es, como ya vimos, la creación de surtido. Si un turista desea visitar un destino (Tenerife, pongamos por caso) en unas fechas determinadas y sólo visita las web de los establecimientos alojativos podría perder horas en Internet para descubrir que casi todos los establecimientos que pretende visitar están ocupados en las fechas que le interesan. Por el contrario, una visita a un intermediario le da, en unos instantes, una visión panorámica de la disponibilidad en un destino. ¿Han de apoyar los poderes públicos mediante subvenciones más o menos encubiertas (publicidad, descuentos en las tasas aéreas, u otras) a las líneas aéreas de bajo coste? Creemos que actuaciones en ese sentido van a favorecer a unos agentes y perjudicar a otros, incluso dentro de un mismo destino turístico. Por lo tanto, pensamos que es necesaria más investigación y más reflexión al respecto antes de tomar decisiones de tanta importancia.

\section{Agradecimientos}

Este trabajo se llevó a cabo en el marco del Máster Universitario en Dirección y Planificación del Turismo de la Universidad de La Laguna, en concreto en su tercera edición (curso 2009-10). Por ello, y en la medida en que el mismo se ha nutrido del valioso ambiente intelectual del máster, queremos mostrar nuestro agradecimiento al alumnado y profesorado del mismo. También hubiera sido imposible la realización de este trabajo sin la ayuda de Turismo de Tenerife y de su responsable, Silvia Canales, que nos facilitó el poder trabajar con los datos de la encuesta a la que aquí se hace referencia. Ceferino Mendaro, de la empresa INVESTUR, aportó su experiencia y conocimiento del medio turístico en Tenerife y nos hizo ver la importancia creciente del fenómeno del turismo residencial "para legal" en algunas zonas de la isla. Por último, la Asociación de Hoteleros de Tenerife, La Palma, La Gomera y El Hierro (ASHOTEL), y en particular Juan Pablo González Cruz, dentro de la misma, nos ayudaron a tener una visión más amplia acerca de cómo, desde el punto de vista empresarial, se ve en la actualidad el tema de la intermediación turística.

\section{Bibliografía}

Alcázar Martínez, Benjamín

2002 Los canales de distribución en el sector turístico.

Universidad de Oviedo. ESIC Editorial

Agarwall, S.

2002 Restructuring seaside tourism: The Resort Lifecyle, en Annals of Tourism Research, 29(1): 25-55

Benjamin, R.I. y Wigand, R.T.

1995 "Electronic Markets and Virtual Value Chains on the information Superhighway", en Sloan Management Review, winter (Pág. 62-72)

Benjamin, R.I. y Wigand, R.T.

1997 "Electronic Commerce: Effects on Electronic Markets", en Journal of Computer Mediated Communication, 1(3).

Butler, R (ed)

2006 The tourism area life cycle: applications and modifications, Channel View Publications, Londres

Cardoso, J.

2006”Developing Dynamic Packaging Systems using Semantic Web Technologies", en Transactions on Information Science and Applications, 3(4): 729-736

Cardoso, J y Lange, C.

2007 A Framework for Assessing Strategies and Technologies for Dynamic Packaging Applications in E-Tourism, en Information Technology \& Tourism, 9(1): 27-44(18)

Cavlek, N.

2005 "El papel de los turoperadores en el desarrollo del turismo internacional. Una evaluación." en Política y Sociedad 42(1): 117-133.

Doherty, N.F, Ellis- Chadwick, F y Hart, C.A

1999 "Cyber retailing in the UK: the potential of the Internet as a retail channel", en International Journal of Retail \& Distribution Management, 27(1): 22-36.

Esteban, A., Millán, A. y Molina, A.

2000 "La Comercialización de Productos Turísticos en Internet: Implicaciones para las Agencias de Viaje”, en Cuadernos de Ciencias Económicas y Empresariales, 39, 15, 35.

Eymann, A. y Ronning, G.

1992 "Discrete Choice Analysis of Foreign Travel Demand" en Vosgerau, H.J., ed., European Integration in the World Economy. Studies in International Economics and Institutions, Berlin: Springer.

Gereffi, G.

2005 "The Global Economy: Organization, Governance, and Development”, en Smelser, N. J. y Swedber, R.: The handbook of Economic Sociology: Second Edition, Nueva York, Princeton University Press 
Falkenstein, H.

1997 "Nuevos Medios y Tecnologías de Distribución en el Sector Turístico. ¿Amenazas u Oportunidades para las Agencias de Viajes Minoristas?", en Estudios Turísticos, 134: 23-34

Hawkins, R; Mansell, R; Steinmueller W.E

1998 "Towards Digital Intermediation", en The European Information Society, FAIR Working Paper, $\mathrm{n}^{\circ} 50$. SPRU. March

Huete, R.

2009 Turistas que llegan para quedarse. Una explicación sociológica sobre la movilidad residencial, Alicante, Publicaciones de la Universidad de Alicante

Ivars Baidal, J. A.

2006 "La expansión de las compañías aéreas de bajo coste: Análisis del impacto en la actividad turística española”, en Congreso de Turismo, Universidad y Empresas, Castellón Mayo 2006.

Mak, J. y Moncur, J.E.T.

1980"The Demand for Travel Agents", en Journal of Transport Economics and Policy, mayo, (Pág. 221231)

Parra López, E., Melchior Navarro, M y Ramos Domínguez, A.

2003 "Análisis e impacto de los touroperadores y las agencias de viaje en el transporte turístico: nuevas tendencias en Canarias", en Pasos, Revista de Turismo y Patrimonio Cultural, 1(2): 217-229, disponible on line en www.pasosonline.org

Parra, Eduardo

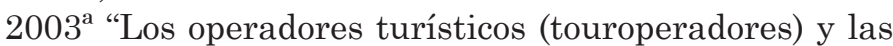
empresas de transportes (agencias de viajes)" En: Empresa y transporte en Canarias. Santa Cruz de Tenerife. Fundación Fyde-CajaCanarias (Pág. 197219)

Parra, Eduardo y otros

2003b "Análisis e impacto de los tour operadores y las agencias de viajes en el transporte turístico: nuevas tendencias en Canarias." En: XVII Congreso nacional, XIII Congreso hispano-francés. Bordeaux. (Pág. 1017-1026)

Rodríguez González, P. y O. Molina Molina

2009 "El comportamiento turístico de los españoles. Segmentación en función de criterios objetivos específicos", en El turismo en el Mediterráneo. Posibilidades de desarrollo y cohesión. M. Latiesa, I. Puertas y J. L. Paniza. Madrid, Ramón Areces: 101-120.

Santana Turégano, M. A.

2009 "Desarrollo turístico y desarrollo socio- económico. Un enfoque desde el nivel regional”, en Revista Brasileira de Docência, Ensino e Pesquisa em Turismo, 1(2): $63-88$
Sheldon, P.J. y Mak, J.

1987 "The Demand for Package Tours: A Mode Choice Model" en Journal of Travel Research, 25(3): 13-17. Turismo de Tenerife

2009 Estadísticas de Turismo Receptivo: Turismo en cifras año 2009 (Turistas alojados en Tenerife por tipología de alojamiento) Fuente: STDE Cabildo Insular de Tenerife

\section{NOTAS}

1. En el caso de Tenerife, al tratarse de un destino insular, la práctica totalidad de los turistas, salvo los internos o aquellos que puedan venir de alguna otra de las islas Canarias llegan a la isla por vía aérea. En realidad, muchos de los destinos de sol y playa más importante son también destinos insulares: Baleares, Islas Griegas, Malta, Chipre, etc. Y otros que no lo son, en la medida en que se especializan en un turismo que podríamos denominar "climático", reciben a sus turistas fundamentalmente por avión, ya que es el único medio de transporte que permite a turistas de climas fríos desplazarse en pocas horas a disfrutar de unos días a lugares de climas cálidos. Sería el caso de la Costa del Sol (España), el Algarbe (Portugal) o Florida (Estados Unidos).

2. Un ejemplo de esto, para el caso español, sería la compañía "Rumbo.es", que ante los requerimientos de un cliente para viajar entre dos puntos distintos ofrecer como alternativa los vuelos de las compañías "Low Cost" más significativas, como Ryanair, Easyjet o Monarch.

3. Un análisis de las web de dos de las compañías "Low Cost" más importantes aporta una interesante información sobre este aspecto. Si contratamos un vuelo a través de Ryanair se nos ofrece, en el mismo proceso de reserva, la opción de reservar un vehículo de alquiler, en este caso con Hertz. En el caso de que queramos también contratar alojamiento, un enlace a la web de "Booking" en la página principal de Ryanair nos permitiría hacerlo, así como también contratar "Airport Transfer". Si hacemos una reserva con Easyjet, al reservar un vuelo se nos ofrece la posibilidad de reservar también un alojamiento primero y después un vehículo de alquiler. Al buscar información en la web nos enteremos que el servicio de reserva de alojamiento es ofrecido por una compañía llamada "LateRooms Limited", compañía con sede en el Reino Unido (igual que Easyjet) y que, tal y como se especifica en la web, es subsidiaria de Tui Travel (más información en http://hotels. easyjet.com/es/p5655/Global/termsofuse/default.aspx . Como podemos ver, en ambos casos, especialmente en el de Easyjet, el cliente puede tener la sensación de estar contratando un "paquete dinámico" que se adapta a sus características, adquiriendo en un mismo proceso los componentes principales 
de un viaje: transporte hasta el destino (avión), alojamiento y transporte en el destino (coche de alquiler). En realidad, detrás de esto se encuentran distintas relaciones entre empresas, que configuran una estructura distinta de la industria turística, donde se podría hablar más que de "desintermediación" de "re- intermediación.

4. Aunque la forma en que se recogieron los datos no permite hacer afirmaciones tajantes al respecto, parece obvio que los turistas con mayor número de visitas a la isla tienen también mayor edad media que los que la visitan por primera vez. Es decir, un turista tiene más probabilidades para haber acudido repetidas veces a un destino cuanta mayor es su edad. Los autores que plantean que el impacto de las NTIC en el turismo conduce a la desintermediación sugieren que en la medida en que, por motivos meramente demográficos, los turistas nacidos a mitad del siglo XX, con nulas o limitadas habilidades en el uso de las NTIC, sean sustituidos por turistas nacidos a finales de dicho siglo, con mayor habilidad en el uso de las mismas, se dará la desintermediación. Este idea se basa en el supuesto de que lo que impide un mayor uso de la contratación directa son las limitadas capacidades informáticas de la población. Como se ve, en este trabajo apostamos por otra hipótesis: lo que resulta determinante es la configuración del sector, y por lo tanto, la tecnología no hace más que adaptarse a la misma.

5. En concreto, la respuesta a la que contestaban los turistas, en la versión española de la encuesta, obviamente, era la de ¿Cómo y con quién contrató el alojamiento que ha utilizado durante este viaje a Tenerife? Y las opciones de respuesta eran "Touroperador", "Agencia de Viajes" o "Alojamiento". Para cada una de esta opciones se podía a su vez marcar la opción "a través de internet" o "Directa/ Personalmente.

6. Las categorías consideradas para el análisis fueron: Hotel 1 y 2 estrellas, Hotel 3 estrellas, Hotel 4 estrellas, Hotel 5 estrellas, Apartotel 1 y 2 estrellas, Apartotel 3 estrellas, Apartotel 4 estrellas, Apartotel 5 estrellas, Apartamentos de 1 llave, Apartamentos de 2 llaves, Apartamentos de 3 llaves, Time Sharing, Casa o apartamento Privado.

7. Así por ejemplo, la web Homeholidays (www.homeholidays. co.uk ), basada en el Reino Unido, ofrece (visita realizada en Septiembre de 2010) 228 apartamentos vacacionales en Tenerife, facilitando los datos de la vivienda y una manera de ponerse en contacto con los propietarios. Esto representa el $44 \%$ de los apartamentos que ofrece en Canarias (518) de un total de más de 8.000 que ofrece en todas España, oferta que se centra sobre todo en aquellos lugares en que más fuerte es la importancia del turismo residencial británico: Cataluña y Andalucía (entorno a un $24 \%$ del total de plazas cada una), Baleares (18\%), C. Valenciana (14\%) y Canarias (6\%).
Recibido:

$04 / 10 / 10$

Reenviado:

$14 / 12 / 10$

Aceptado:

$07 / 01 / 11$

Sometido a evaluación por pares anónimos 